\title{
Expansión metropolitana de la ciudad de Managua
}

\section{Metropolitan expansion of the city of Managua}

\author{
Gutiérrez Lanza, Danna ${ }^{1}$ \\ ${ }^{1}$ Investigadora Independiente, Becaria Fulbright/Laspau- Universidad \\ del Estado de Michigan, Estados Unidos \\ 1danna.gutierrez@doc.uca.edu.ni/https://orcid.org/0000-0003-3090-7853 \\ Recibido el 15 de noviembre de 2021, aprobado el 20 de diciembre de 2021
}

RESUMEN | Este artículo indaga sobre los factores y características del fenómeno de expansión urbana a fin de encontrar en las cualidades la lógica explicativa al desarrollo urbano más allá de la dimensión física del mismo. Los resultados permiten caracterizar una expansión a nivel metropolitano que comparte características de desarrollo espacial con el resto de las ciudades en Latino América, y que encuentra su impulso en un modelo de urbanismo neoliberal, a saber: acelerado crecimiento suburbano, caracterizado por su baja densidad y su extensión más allá de los límites urbanos.

La transferencia de facto de las capacidades de gestión desde las entidades estatales hacia el mercado como agente primario del desarrollo urbano han dado como resultado una expansión urbana horizontal que profundiza las desigualdades urbanas y las inequidades sociales en una ciudad que cada vez más se caracteriza por un desarrollo fragmentado de enclaves funcionales y llevado hacia adelante a través de la inversión en innovaciones espaciales.

En Nicaragua, específicamente en la ciudad de Managua, es posible observar cómo estas condiciones consolidan la tendencia al aislamiento y la fragmentación del tejido urbano. La inversión privada sobre ejes viales y a través de proyectos habitaciones como "innovaciones espaciales" avanzan a través de la habilitación de tierra no urbanizada, incidiendo en cambios en la relación funcional entre los centros urbanos y entre estos y las zonas habitaciones en las áreas interurbanas.

PALABRAS CLAVE | Áreas Metropolitanas, Expansión Urbana, Managua, Proyectos Habitacionales 


\begin{abstract}
This article looks into the factors and characteristics of the phenomenon of urban growth in order to find in the qualities of the process the explanatory logic to urban development beyond its physical dimension. The results allow characterizing an expansion at the metropolitan level that shares characteristics of spatial development with the rest of the cities in Latin America, and that finds its impetus in a neoliberal urbanism model, namely: accelerated suburban growth, characterized by its low density and its extension beyond the urban limits.
\end{abstract}

The de facto transfer of management capacities from state entities to the market as the primary agent of urban development has resulted in a horizontal urban expansion that deepens urban inequalities and social inequalities in a city that is increasingly characterized by a fragmented development of functional enclaves and carried forward through investment in spatial innovations.

In Nicaragua, specifically in the city of Managua, it is possible to observe how these conditions consolidate the tendency to isolation and fragmentation of the urban fabric. Private investment on road axes and through residential projects such as "spatial innovations" advance through the habilitation of undeveloped land, influencing changes in the functional relationship between urban centers and between these and residential areas in interurban areas.

KEYWORD | Metropolitan Areas, Urban Growth, Managua, Housing Projects

\title{
1. Introducción
}

Sí bien la ciudad de Managua no evoca la imagen de una gran urbe metropolitana, no se puede pasar por alto que en la capital nicaragüense se están desarrollando, desde hace varios años, una serie de características físico-espaciales que remiten a los inicios de la conformación de un área metropolitana, o que en palabras de Eduardo Rojas (2005) estamos ante una "ciudad con rasgos de área metropolitana" (p.35).

Las estrategias y planes desarrollados para el área metropolitana de Managua - la llamada propuesta mexicana de 1973; el Plan de Acción para la Región Metropolitana de la Alcaldía de Managua en 2007 que la constituía por 29 municipalidades; la definición hecha por el Banco Interamericano de Desarrollo en el Plan de Acción Managua Sostenible en 2013, y el más reciente esfuerzo de la Alcaldía de Managua en 2019 denominado Plan Integral para la Conformación del Área Metropolitana de Managua, según lo nombra la recientemente publicada Cartilla Urbanística de la Alcaldía de Managua (2021) - son esfuerzos que sí bien no han tenido éxito en institucionalizar el área metropolitana, su existencia es sintomática de cómo, desde las instancias municipales, se perciben los cambios en el carácter espacial del área urbana y la necesidad de su gestión.

Este artículo se enmarca en una investigación más amplia, y sí bien se inicia considerando la trayectoria de esfuerzos institucionales y presenta un esquema explicativo del concepto de área metropolitana, su objetivo no es ofrecer un camino hacia la metropolización de Managua, sino de analizar los antecedentes de este proceso en un marco de desarrollo urbano más allá de las características físicas. Los resultados aportan a la discusión teórica, informada por la 
investigación empírica, sobre la caracterización de una expansión a nivel metropolitano que comparte características de desarrollo espacial con el resto de las ciudades en Latino América, y que encuentra su impulso en un modelo de urbanismo neoliberal, a saber: acelerado crecimiento suburbano, caracterizado por su baja densidad y su extensión más allá de los límites urbanos. Este avance en la discusión teórica permitiría a su vez, cuestionar y mejorar las políticas urbanas que habilitan el desarrollo de nuestras ciudades.

Así, se tiene como objetivo principal:

- Analizar el proceso de urbanización a escala metropolitana en la ciudad de Managua como fenómeno multidimensional.

Y como objetivos específicos:

- Identificar los factores y características del patrón de crecimiento espacial de Managua.

- Describir las cualidades del proceso de expansión urbana.

En su totalidad, este artículo se compone de 6 secciones, incluyendo esta introducción, en la que se presentan la fundamentación teórica que sirve para el análisis de los resultados obtenidos, la discusión de estos resultados, y las conclusiones para terminar con una serie de recomendaciones sobre las líneas de investigación que se abren a partir de estos hallazgos iniciales.

\section{Metodología}

Este artículo presenta resultados a nivel exploratorio, basados en la recolección, relevamiento y procesamiento de una revisión documental, en el marco de una investigación más amplia. El enfoque metodológico de la investigación es cualitativo, de alcance descriptivo y de corte longitudinal.

La escasez de información constituye una importante limitación para el análisis, a la cual se hace frente a través de la sistematización de datos que se encuentran disgregados en mapas oficiales, y que se han complementado a través de información extraída de fuentes satelitales de libre acceso, y datos censales de fuentes secundarias. Esto además permite una primera aproximación a la cuantificación del fenómeno, cuando el análisis así lo requiere.

Se analiza, de manera cualitativa, el crecimiento de la ciudad a través del estudio de la mancha urbana, utilizando imágenes satélites de código abierto, que permiten ubicar y trazar los contornos de los proyectos habitacionales que llevan adelante la expansión de la ciudad, como se discute más adelante, y cuyo resultado es el mapa que se presenta en la sección de resultados. Adicionalmente, se hace recuento de la información disponible de 20 años de desarrollo urbanístico en la ciudad de Managua, entre 1998 - 2018.

\section{Desarrollo}

Según Tomás (2016) "el proceso de urbanización en el mundo es una tendencia estable y en aumento", y la región de América Latina y el Caribe demuestra esta tendencia albergando "el 13\% de la población urbana y el 14\% de áreas metropolitanas" (p.3). Sin embargo, como es bien sabido, el proceso de urbanización vertiginoso que ha experimentado la región también ha traído consigo grandes desigualdades sociales y problemas medioambientales. 
En una línea de análisis sociológico Touraine (s.f.) va a firmar que la ciudad se encuentra en un proceso de transformación, tanto física y morfológica, como funcional y categórica. Todas las ciudades en su multiplicidad de funciones y aspectos componentes no se presentan como una unidad que avanza al unísono; en ocasiones los aspectos económicos entran en auge a expensas de la decadencia de las virtudes sociales y culturales.

\section{¿Conurbación o metropolización?}

La primera interrogante que surge respecto a esta transformación a la que alude Touraine y que Tomás relaciona con las áreas metropolitanas es la definición misma de área metropolitana. Tello y Robledo (2011) proponen entender estas áreas como unidades de articulación territorial, y citan a Pedro Pírez al referirse a la característica de heterogeneidad de las actividades y grupos sociales que engloban la forma metropolitana. Hasta aquí, el concepto sigue siendo difuso en tanto no parece alejarse mucho de la conceptualización que pudiéramos tener de las ciudades mismas.

El punto de partida para esclarecer el término es, posiblemente, la definición del proceso de conurbación, que el Centro Iberoamericano de Desarrollo Estratégico Urbano CIDEU (s.f.) define como

una región eminentemente urbana surgida de la expansión de varias ciudades próximas entre sí y que pueden ser de diverso tamaño, aunque una de ellas suele ser mayor que el resto. Dichas ciudades se integran en un sistema, aunque las partes mantengan su independencia funcional y dinámica (p.3).

Entonces bien, la conurbación se refiere a un proceso que puede entenderse como natural o propio del proceso de expansión de ciudades que se encuentran próximas unas a otros, y que eventualmente ven sobreposición en sus territorios, tanto en los delimitados administrativamente como estados, municipios, comarcas, etc., como en la influencia que los núcleos urbanos ejercen (ver figura 1).

Figura 1. Esquema ilustrativo del proceso de conurbación y metropolización de las áreas urbana

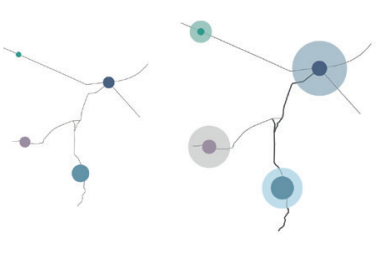

Expansión Urbana

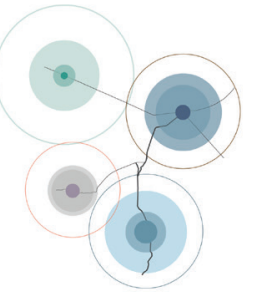

Conurbación

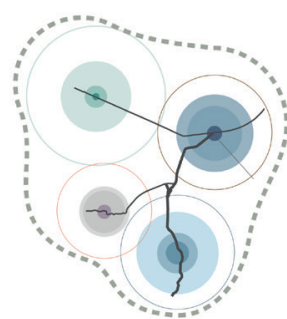

Institucionalización

Nota. Elaborada por la autora, 2021

En esta suerte de proceso, el siguiente paso es la conversión de la conurbación en un área metropolitana, que de forma general tiene lugar cuando esta "se convierte en una entidad de orden estratégico (...) y se activa la capacidad de relacionarse y coordinar voluntades y políticas con visión de conjunto" (CIDEU, p.3). Por lo tanto, podemos entender el área metropolitana como una modalidad institucionalizada de la conurbación de ciudades. Aunque aquí cabe destacar que existen diversas modalidades de institucionalización, sin lugar a duda, más allá de la velocidad y cualidad de la conurbación o de la modalidad de institucionalización, estos procesos implican una reestructuración de los territorios urbanos. 


\section{Metropolización en Latinoamérica: Características del patrón espacial}

Al hablar de Latinoamérica y el Caribe, Ciccolella (2012) señala el agravamiento de las desigualdades sociales y económicas, las cuáles además tienen una dimensión territorial. La región parece estar viendo en mayor o menor medida la consolidación del "modelo de ciudad anglosajónamericano, más disperso y más segmentado, y estructurado en 'islas', conectadas a través de redes de autopistas" (p.10). Si bien el autor reconoce que existen singularidades en el desarrollo de todas las ciudades, cabe rescatar su planteamiento en relación con las semejanzas que identifica en las principales urbes metropolitanas de la región. En términos socioespaciales, Ciccolella (2012, p.13) nombra:

i. Expansión de la mancha urbana, ii. Policentrismo o, al menos, tendencias al mismo, iii. Tendencias a la ciudad-región y al crecimiento reticular, iv. Expansión de la base económica, especialmente basada en servicios, v. Difusión o proliferación de nuevos objetos urbanos (shoppings, hipermercados, urbanizaciones cerradas, edificios inteligentes, etc.), vi. Suburbanización difusa, tanto de élites como de sectores pobres o populares, vii. Incremento de la polarización social, viii. Incremento o consolidación de la segregación residencial, ix. Aparición de nuevos distritos de negocios o formación de redes de distritos corporativos

Estas características físicas, como la expansión de la mancha urbana y la tendencia a una suburbanización difusa, las amplia Rojas (2005), quién además destaca algunas problemáticas que resultan del fenómeno, al resaltar cómo ese "crecimiento periférico suburbano de baja densidad $\mathrm{y}$, muchas veces, discontinuo, extiende el área urbanizada más allá de los límites de los términos municipales" (2005, p.38), y resulta en problemáticas bien estudiadas como la baja densidad de esta expansión y la implicación a extender redes de infraestructura de servicios, que encarece los costos de vivir en la ciudad.

En este trabajo, la propuesta es estudiar el fenómeno y particularmente esas características de la expansión a través del marco de análisis que propone Brites (2017), en el que se entienden estas mismas como el resultado del entrecruce de estrategias de desarrollo urbano que han caracterizado a las ciudades latinoamericanas desde la década de 1990. Destaca así:

a) la inversión selectiva de obras públicas en áreas urbanas; b) la implementación de nuevas legislaciones de ordenamiento y renovación urbana; c) la generación de entornos «embellecidos» y la promoción de competitividad turística; d) el facilitamiento al mercado inmobiliario para emprendimientos residenciales, comerciales, etc., e) la implementación de políticas habitacionales en áreas de apertura urbana o periféricas (p. 574).

Lo que interesa más de estas estrategias enumeradas, y de la propuesta del autor, es el análisis de la expansión urbana más allá de las características físicas del fenómeno, o de estas como síntomas de procesos urbanos de orden más amplio. Esta aproximación nos permite conectar con otras ideas que han tomado fuerza en la literatura latinoamericana para encontrar la lógica que da explicación a estos procesos.

Mientras que un análisis de las consecuencias de este fenómeno queda fuera de los alcances delimitados en esta investigación, no está de más señalar en el contexto algunas de ellas. Tal cómo afirma Tomás (2016) las desigualdades sociales y las problemáticas medioambientales son los grandes problemas que tienen en su origen, aunque no son exclusivos de, la expansión horizontal de las ciudades.

La conformación de entornos urbanos extensos, disgregados y fragmentados genera ciudades 
altamente segregadas y divididas. El fenómeno ha producido una "urbanización de la pobreza" en las ciudades latinoamericanas (Rojas, 2005), donde la disparidad y desigualdad en la distribución de bienes, servicios y de acceso a las oportunidades crea una segregación espacial que se corresponde con una alta fragmentación social, permitiendo así la continuidad temporal de problemáticas más amplias como la reproducción intergeneracional de la pobreza.

\section{Una propuesta explicativa de la urbanización metropolitana}

Siguiendo la propuesta de Brites podemos proponer entender la producción de la ciudad hoy a través de una matriz que cruza actores, proyecto y políticas o legislación urbana para habilitar la producción de las ciudades desde un modelo neoliberal, en el que cobra fuerza el mercado como actor y constructor y constructor, y la oferta-demanda como política.

Este esquema de desarrollo urbano estaría detrás de la expansión física de las ciudades, siendo el motor de los crecimientos dispersos y fragmentados. Para Ciccolella (2012) la habilitación de este modelo tiene lugar en los cambios estructurales de los modelos de desarrollo que han optado por flexibilizar el rol de las entidades estatales y darle primacía al avance del capital. Este último termina incidiendo, sino controlando, los procesos de decisión y ejecución de los proyectos en el territorio urbano. Brites (2017) propone entender este modelo de producción de la ciudad desde un marco analítico de desigualdad urbana, planteamiento que está de acuerdo con propuestas de análisis del mercado del suelo urbano como las de Abramo (2012).

Los trabajos de estos autores describen desde sus distintas perspectivas el fenómeno de urbanización a escala metropolitana que se ve en las ciudades latinoamericanas y que Abramo (2012) describe como una estructura espacial fraccionada. En la propuesta de este último autor encontramos una aproximación interesante para explicar el fenómeno de la expansión metropolitana, desde un análisis crítico del modelo de desarrollo urbano de ciudad neoliberal, como lo llama Brites.

En un modelo que ha llamado de "ciudad com-fusa" Abramo (2012) describe el funcionamiento de los mercados de suelo formal e informal en América-Latina, en el que la existencia de ambos mercados se entiende como simultánea y no mutuamente excluyente. Abramo describe cómo los mercados altamente segmentados desarrollan proyectos - en muchas ocasiones habilitando nuevo suelo urbano - con la intención de crear nuevas ofertas que cumplan con las exigencias del grupo de demanda solvente, mientras que, a través de una desvalorización ficticia, se habilita la adquisición del stock para los sectores socioeconómicos inmediatamente inferiores. En esta lógica, es la búsqueda que hace el mercado inmobiliario, de una externalidad altamente valorizada lo que empuja el desarrollo y la expansión de las ciudades. De modo que es esa "innovación espacial", como lo llama Abramo, lo que promueve el componente de estructura difusa de la ciudad hacia áreas suburbanas. Una estructura que eventualmente configura las ciudades a escala metropolitana, conectando distintos centros urbanos que siguen esta lógica de expansión.

Rodríguez \& Abramo (2005) aseguran que el modelo de grandes proyectos urbanos pretende "intervenir para impulsar el crecimiento económico y apoyar el desarrollo de nuevas funcionalidades" (p.3). Así, una de las características más dominantes de esta estrategia de inyección de capital es su ubicación en inversiones focalizadas y muy selectivas, que cada vez más implican la búsqueda de áreas suburbanas o áreas urbanas no desarrolladas en un intento de las que las inversiones revaloricen áreas con el fin de rentabilizar el capital.

Sobre las propuestas de Abramo (2011) reflexiona Brites (2017) y asegura así que el resultado 
es "el mercado como mecanismo de coordinación de producción de la ciudad", transformando el desarrollo urbano a través de "nuevas materialidades urbanas inclinadas más al consumidor que al ciudadano" (Brites, 2017, p.575) lo que propone entender como un nuevo orden socioespacial.

\section{Resultados}

La llamada Área Metropolitana de Managua, que se ha constituido de facto, pues no existe una ley o instrumento que la decrete o regule, muestra las características específicas de una conurbación en Latinoamérica: la macrocefalia urbana con la primacía amplia de Managua por sobre las siguientes ciudades más grandes e importantes (Antillón, 2013), la formación de inter-espacios urbanos degradados y el asentamiento de grupos en situación de vulnerabilidad en algunas zonas periféricas y la incorporación de áreas rurales donde la continuidad física no es tan densa. Entonces sí bien no nos podemos referir a un proceso de metropolización como formalización para la gestión del área, es indispensable conocer el proceso de conurbación que tiene lugar en Managua.

La ciudad de Managua domina la unidad conurbana del país y en torno a ella se integran las concentraciones de los municipios correspondientes a los departamentos de Carazo, Masaya, Granada y Managua.

Las propuestas hechas para la delimitación geográfica del Área Metropolitana de Managua (también llamada Región Metropolitana de Managua) siempre han correspondido a la relación funcional directa que se ha establecido entre la ciudad de Managua y los núcleos urbanos más próximos a razón de su atracción como polo de concentración de servicios gubernamentales y de comercio y su capacidad instalada, por ejemplo, para la generación de empleo. Estas relaciones funcionales se manifiestan en intercambios a nivel económico y social, y se hacen evidentes en la forma física del territorio y las manchas que se expanden sobre las principales vías, conectando las áreas suburbanas de un municipio con los centros poblacionales del siguiente. El área se puede estudiar inicialmente estableciendo la relación entre 9 municipios: Managua como centro (por su primacía urbana), Tipitapa, Mateare, Ciudad Sandino, Ticuantepe, El Crucero, Nindirí, Masaya y La Concepción. La conurbación de estos se explica por, al menos, los siguientes factores.

\section{Cómo y cuánto crece y se expande el entorno conurbado}

En Managua, especialmente a partir del terremoto del 1972, el modelo de crecimiento se ha caracterizado por su baja densidad y su expansión hacia áreas suburbanas bajo un patrón disperso que avanza sobre los ejes viales radiales que conectan la ciudad con los municipios aledaños, especialmente hacia el suroeste (Gutiérrez, 2020) (ver figura 1). Estas se han convertido en ejes importantes de inversión privada para la construcción de edificios comerciales y empresariales, y las viviendas de la "clase media y alta" (Rodgers, 2006). Este crecimiento se ha dado "sin procesos de planificación, por lo cual la expansión se dio de forma descontrolada, en bajas densidades, con altos consumos de suelos rurales y suburbanos, y sin las provisiones necesarias en infraestructura y servicios básicos" (BID, 2013, p.51).

Este crecimiento horizontal, virtualmente desregulado ha implicado el aumento de la mancha urbana de la ciudad en un $24.3 \%$ aproximadamente en los últimos veinte años, según la publicación del BID. Las modalidades de expansión de la ciudad, residenciales cerrados y asentamientos informales, alrededor de los ejes viales principales hacia el sureste, sobre todo la carretera a Masaya, crean una concentración de usos mixtos sobre el eje y expande la mancha de forma lineal y paralela a esta, hasta casi conectar con el núcleo urbano de Masaya, con una densidad en aumento. Fenómeno que se ha visto acrecentar en las carreteras Norte, Sur, Vieja hacia León y Nueva hacia 
León en años más recientes.

Figura 2. Mancha urbana de la ciudad de Managua al 2005 y al 2005
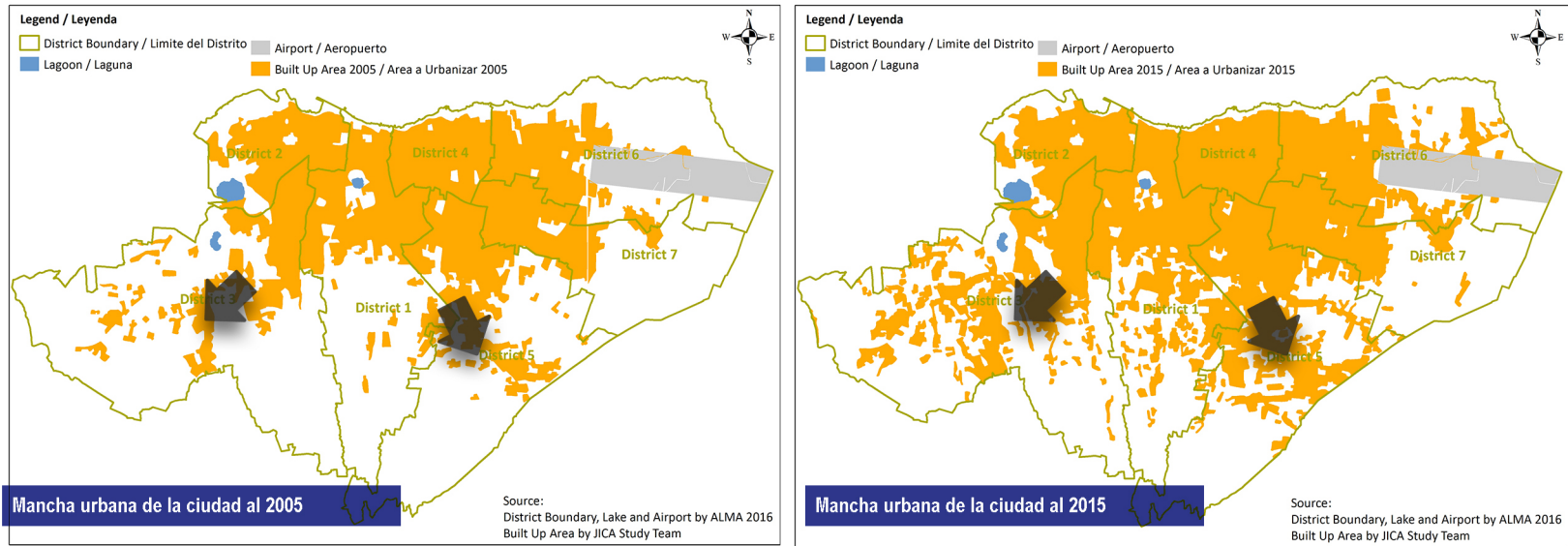

Nota. Plan de Desarrollo Urbano Municipal para la ciudad de Managua, JICA (2017)

La figura 2 permite analizar los mapas presentes en el Plan Maestro para el Desarrollo Urbano del Municipio de Managua, que muestra cómo la mancha urbana entre 2005 y 2015 (mapas en la parte superior de la figura) se consolidaba al interior al tiempo que se expandía sobre los ejes viales ya mencionados.

Figura 3. Expansión de la mancha urbana según zonificación vigente (Áreas Urbana, Suburbana y Rural; y Zonas de Protección y Zonas de Reserva Natural)

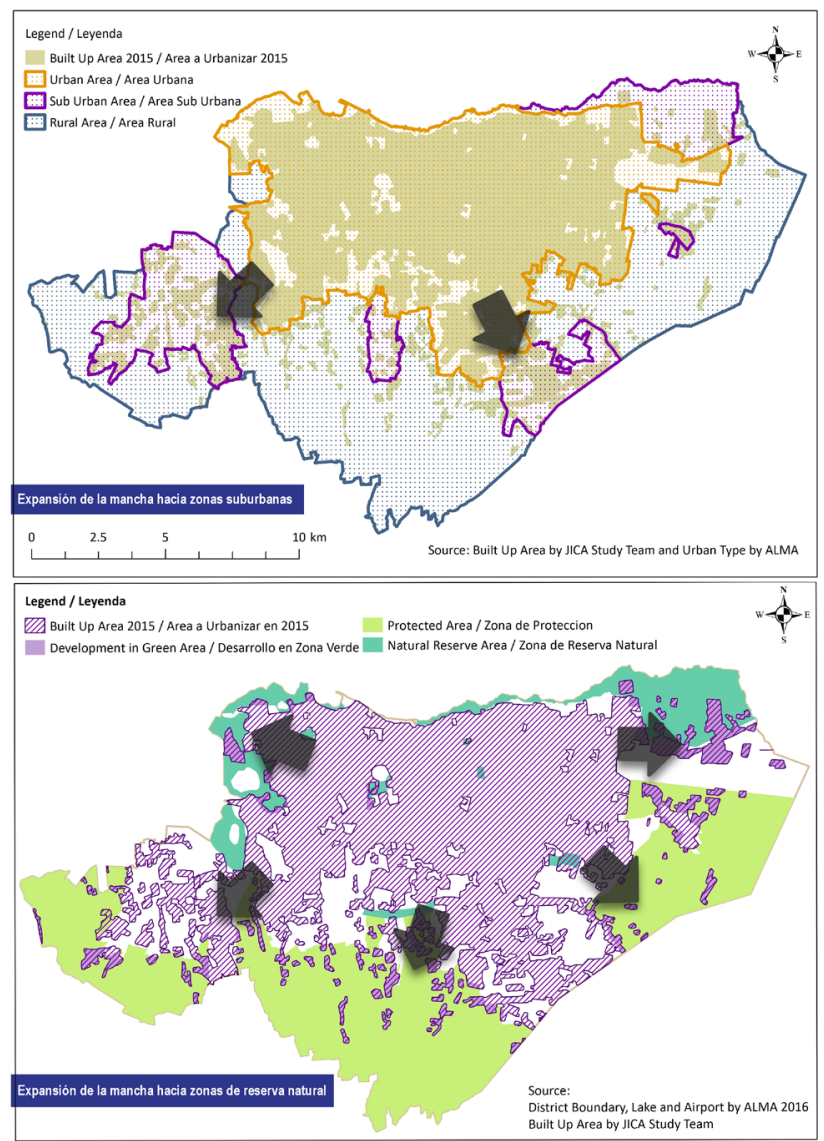

Nota. Adaptado de Plan de Desarrollo Urbano Municipal para la ciudad de Managua, JICA (2017) 
Más allá, los mapas siguientes que se exponen (Expansión hacia Zonas Suburbanas al centro, y Expansión hacia Zonas de Reserva Natural, parte inferior) muestran algunas de las cualidades más importantes de la expansión a nivel metropolitano en Managua, a saber: la expansión horizontal sobre las zonas suburbanas, incluso zonas de reserva natural, y al mismo tiempo dan luz al proceso de habilitación de suelo para urbanizar que está teniendo lugar en la ciudad.

\section{El grado de autonomía, jerarquía y dependencia de los núcleos urbanos conurbados}

Managua es la capital de la república y sede central del poder, además de albergar las sedes centrales de las entidades proveedoras de servicios básicos. Los polos de atracción de empleo, educación, bienes y servicios especializados también se centran en la ciudad. Esta es además la puerta de entrada de turistas extranjeros, pues en ella se ubica el único aeropuerto internacional, que luego conectan por vía terrestre a los destinos destacados del país, entre los que se incluyen ciudades como Granada y Masaya por su atractivo cultural.

Las ciudades satélites que se agrupan y organizan alrededor de la capital son núcleos urbanos dependientes de las funciones centrales que se albergan en Managua. El grupo de cooperación japonesa para el proyecto del Plan Maestro de Desarrollo Urbano calcula el número de pasajeros que utilizan la terminal de buses UCA (de dónde salen los buses intermunicipales a las ciudades más cercanas conectadas en la RMM) en un promedio de 28,600 al día. El hecho remite a la predominancia de movimientos pendulares entre Managua (trabajo) y las otras ciudades, como Granada, Masaya, Carazo (residencia), o a las áreas desarrolladas que se ubican en los espacios inter-urbanos. La figura 2 muestra este fenómeno de movimiento pendular desde la ciudad después de la jornada laboral, la fotografía muestra el tráfico de salida de Managua (a la izquierda) durante la hora pico después de la jornada laboral $(5: 00 \mathrm{pm}-7: 00 \mathrm{pm})$, mientras el tráfico de entrada a la ciudad (a la derecha) es casi nulo.

Figura 4. Fotografía de la Carretera hacia Masaya (vista sur-norte).

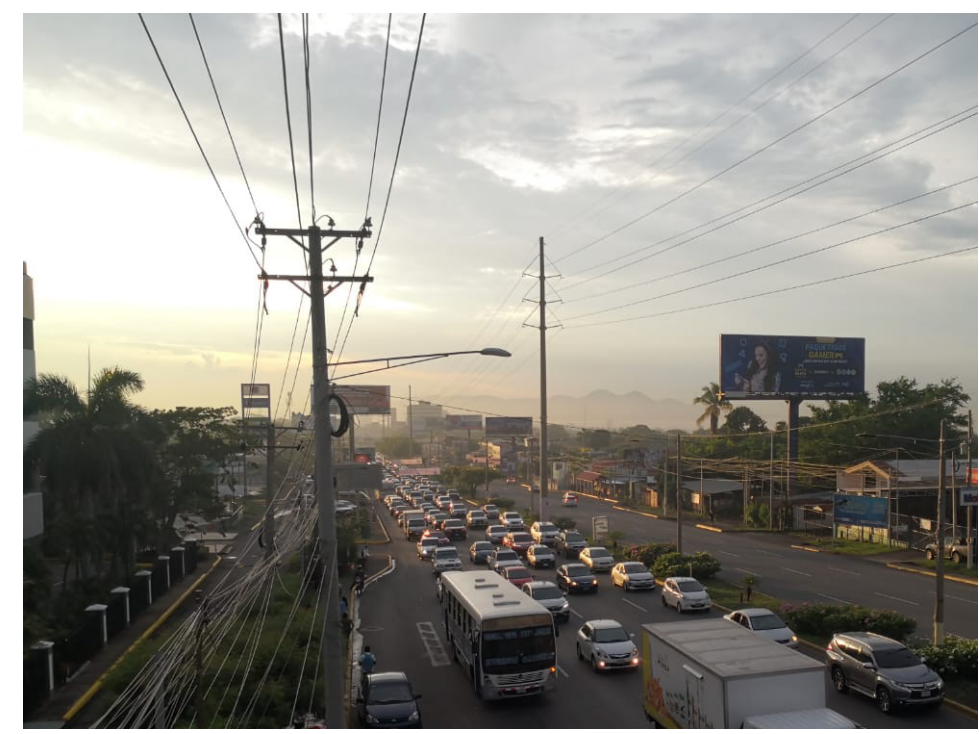

Nota. Pérez Vázquez, R., 2021

\section{Densidad de la población y su reparto sobre el territorio conurbado}

Según datos del Plan de Acción para la Región Metropolitana de ALMA 2007, los municipios que conformarían la región metropolitana sumaban en su territorio al 37\% de la población total 
del país, en una extensión de 6,242.43 km2, con una densidad resultante de 301 habitantes por kilómetro cuadrado. Los municipios cuya tasa de crecimiento superaba la media intercensal se ubicaban alrededor de la ciudad capital (ver tabla 1).

Tabla 1. Proporción de población urbana en el Área Metropolitana de Managua. JICA, 2017

\begin{tabular}{lcccc}
\hline Municipio & Población Total & $\begin{array}{l}\text { Población } \\
\text { Urbana }\end{array}$ & $\begin{array}{l}\text { Población } \\
\text { Rural }\end{array}$ & $\begin{array}{l}\text { Proporción de } \\
\text { población urbana }\end{array}$ \\
\hline $\begin{array}{l}\text { Total Área } \\
\text { Metropolitana }\end{array}$ & 2122316 & 1909246 & 213070 & $90 \%$ \\
\hline Managua* & 1495385 & 1431566 & 63819 & $96 \%$ \\
\hline Tipitapa & 140569 & 127618 & 12951 & $91 \%$ \\
\hline Mateare & 50037 & 46639 & 3398 & $93 \%$ \\
\hline Ciudad Sandino & 112324 & 110083 & 2241 & $98 \%$ \\
\hline Tícuantepe & 36103 & 15495 & 20608 & $46 \%$ \\
\hline El Crucero & 15477 & 9309 & 6168 & $60 \%$ \\
\hline Nindiŕ́ & 54573 & 25866 & 28707 & $47 \%$ \\
\hline Masaya & 176345 & 125825 & 50520 & $71 \%$ \\
\hline La Concepción & 41503 & 16845 & 24658 & $41 \%$ \\
\hline
\end{tabular}

Nota. Basado en estimaciones propias y otras ciudades de "Población Total Municipal Estimada Al 30 de Junio, Período 2012-2025” INIDE

Como muestra la tabla 1 Managua concentra cerca de 1.5 millones de habitantes, y su población urbana alcanza el 96\% del total. Según los datos analizados por JICA (2017) y BID (2013) esta representa aproximadamente el $29 \%$ de la población urbana a nivel nacional, aunque su densidad demográfica de 38.5 habitantes por hectáreas es una proporción relativamente baja, casi la mitad de lo que registran otras ciudades centroamericanas.

Cabe agregar que los datos registrados en el Censo 2005 y las estimaciones poblacionales de la Alcaldía de Managua a enero 2016 la tasa de crecimiento poblacional de la ciudad de Managua representa un aumento de $3.87 \%^{1}$ (JICA, 2017). Sí se considera en paralelo la mancha urbana consolidada en ambos períodos ( 2005 y 2015, según los datos presentados por la agencia), un análisis de las imágenes satelitales correspondientes, la superficie urbanizada de la ciudad experimenta crecimiento a una tasa del $4.05 \%$ (ver tabla 2 ).

Tabla 2. Tasa de Crecimiento de la población de Managua y de la mancha urbana.

\begin{tabular}{lccc}
\hline & Población Managua & Mancha Urbana \\
\hline 2005 & $937,489.00$ & 2005 & $93,798,636.28$ \\
\hline 2016 & $1,495,385.00$ & 2015 & $134,116,841.80$ \\
\hline Tasa de crecimiento & $3.87 \%$ & & $4.05 \%$ \\
\hline
\end{tabular}

Nota. Inormación retomada de JICA, 2017 y estimaciones propias a partir de imágenes satélitales

Las estimaciones se alinean con los reportes del Banco Mundial (2017) acerca de la región Centroamericana, donde la tendencia es el aumento de la superficie total urbanizada que resulta

1 Dato estimado por JICA (2017), considerando los índices de error y la divergencia entre las mediciones comparadas 
en zonas urbanas que crecen más rápido que la población urbana. Según el Banco Mundial, en Centroamérica la media de superficie urbanizada per cápita alcanzaba en 2017 los $120 \mathrm{~m} 2$.

Para Managua es posible ilustrar este crecimiento de la superficie urbanizada a través de los datos de mercado inmobiliario, el cual para el año 2016 vendió aproximadamente 4,800 viviendas nuevas (El Economista, 2017) y proyectaba para el año 2017 la venta de unas 5 mil viviendas más, en una tendencia de crecimiento constante, que por supuesto, implica la expansión física de la ciudad. Una contabilización inicial de los proyectos se presenta en el siguiente acápite.

\section{Los proyectos habitacionales como "innovaciones espaciales"}

Los factores descritos anteriormente destacan las características físicas de la expansión metropolitana. Un punto de partida importante, considerando que uno de los síntomas más visibles de esta expansión metropolitana en Managua es precisamente la expresión física del fenómeno a través del crecimiento horizontal sobre los ejes viales, que expanden la mancha urbana de la ciudad. Esta expansión se compone de distintas tipologías, sin embargo, es el desarrollo de la oferta habitacional el que lo caracteriza, y el que se estudia en esta investigación.

Entre 1998-2018 al menos 196 nuevos proyectos de tipología residencial en la ciudad, como muestra el recurso Mapa de Desarrollos Urbanos Habitacionales ${ }^{2}$, publicado por la Alcaldía de Managua. Un recuento utilizando imágenes satelitales permite ampliar la información encontrada en el mapa publicado y muestra la extensión urbana más allá de los límites municipales (ver figura $5)$.

Figura 5. Tasa de Crecimiento de la población de Managua y de la mancha urbana

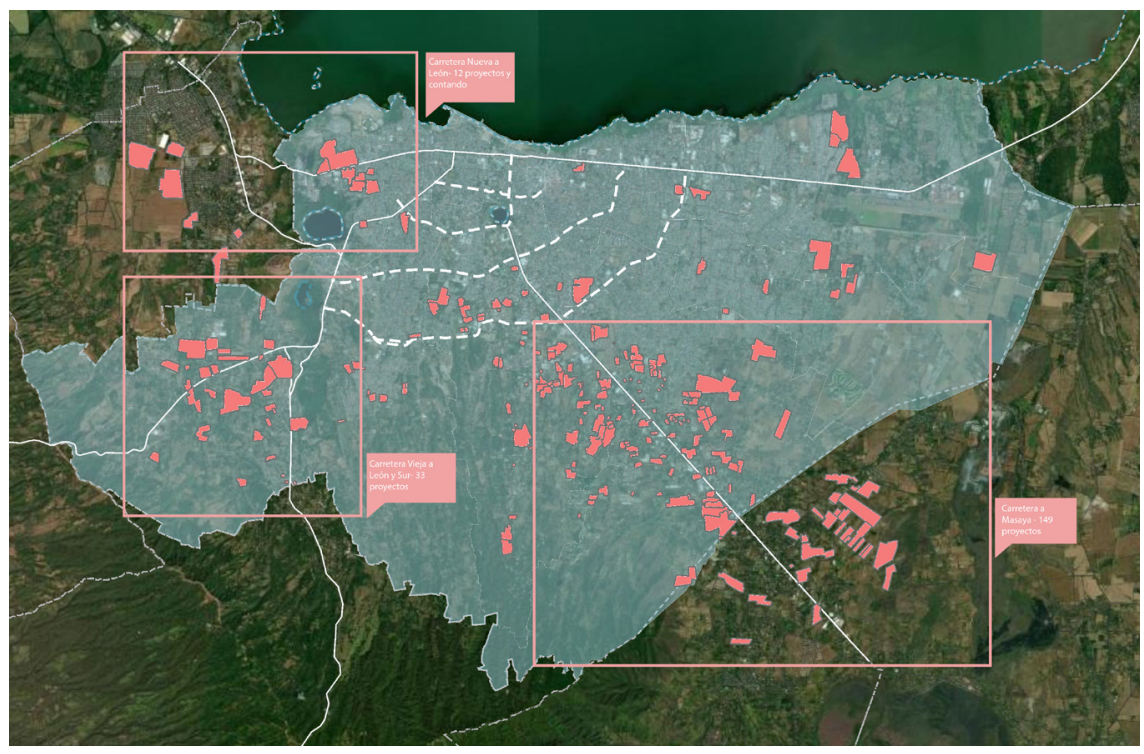

Nota. Elaborada por la autora (2021) con base al recurso Mapa de Desarrollos Urbano Habitaciones

Los proyectos de desarrollo habitacional privado constituyen una inyección de capital importante en la ciudad, lo que ha resultado en una acentuación del proceso de urbanización desordenado, discontinuo y desigual en Managua, mismo que ha generado desigualdades urbanas "caracterizadas por la separación o diferenciación de modos de vida de ciertos sectores sociales" (López, 2016, p.28) con respecto a otros. Un recuento de estos proyectos por año (considerando el año en el que

2 Extraido de https:/www.urbanismomanagua.gob.ni/wp-content/uploads/2020/06/Desarrollos-Habitacionales-40-20-a\%C3\%B1os.pdf

E-ISSN: 2518-2943 | WEB: www.camjol.info/index.php/arquitectura | DOI: https://doi.org/10.5377/arquitectura.v6i12.13102 | E-MAIL: rvarqui.mas@farq.uni.edu.ni 
Figura 6. Proyectos habitacionales en Managua, 1998-2018

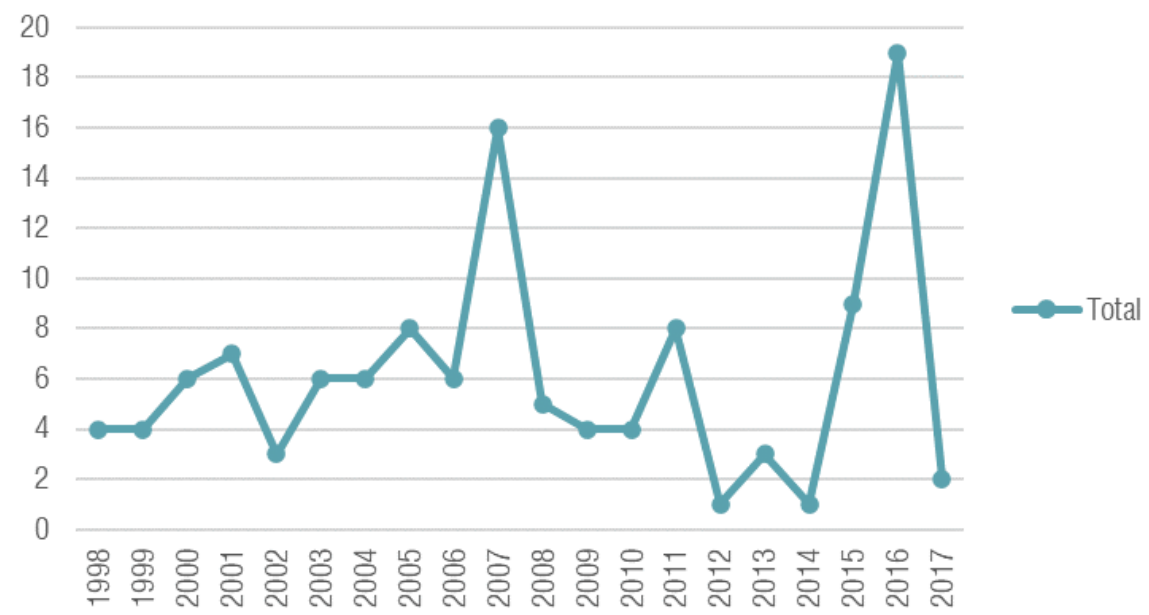

Nota. Elaborado por la autora (2021) con base a datos de ALMA

comienzan a aparecer disponibles en el mercado) (ver figura 6), muestra un desarrollo relativamente sostenido.

Al respecto, Rodgers (2006) describe

[...] un proceso más ponderado de intervención a favor de las elites urbanas, que no ha buscado simplemente sobre imponer una nueva forma urbana sobre las del pasado, sino reestructura activamente el perfil global de la ciudad mediante la separación explícita de ciertos espacios urbanos (p.11).

Para este autor la transformación desigual de la ciudad se torna más evidente a través de una serie de intervenciones o proyectos urbanos a gran escala que tuvieron lugar en la ciudad durante la década de los 90 e inicios del 2000, en lo que él propone entender cómo "un patrón que sugiere una construcción o reconstrucción bastante selectiva" (Rodgers, 2006, p.17). Así, en Managua se comienza a constituir "un archipiélago de enclaves fortificados" conformado por las zonas de residencia de la élite urbana, los puntos de servicio u ocio, que, por su dispersión en el ámbito espacial de la ciudad, hicieron necesaria una red al servicio de la conexión de estos espacios, para otorgar la misma 'seguridad' de las estancias fortificadas a las rutas de desplazamiento.

La inversión en proyectos habitacionales privados en estas áreas que se expanden fuera del límite urbano de la ciudad da forma al proceso de urbanización metropolitana que describe Rojas (2005), acentuando para el caso la proliferación de nuevos objetos urbanos y una suburbanización difusa, que además incrementa al tiempo que consolida la segregación espacial.

La transferencia de facto de las capacidades de gestión desde las entidades estatales hacia el mercado como agente primario del desarrollo urbano han dado como resultado una expansión urbana horizontal que profundiza las desigualdades urbanas y las inequidades sociales en una ciudad que cada vez más se caracteriza por un desarrollo fragmentado de enclaves funcionales $\mathrm{y}$ llevado hacia adelante a través de la inversión en innovaciones espaciales.

\section{Conclusiones}

En una ciudad que muestra baja densidad poblacional, una marcada tendencia al aislamiento y la fragmentación del tejido urbano existente ha tomado fuerza la inyección de capital a través del 
fomento a la inversión privada, mostrados aquí a través de los proyectos habitacionales. Se pueden identificar una serie de dinámicas y estrategias que cruzan la intervención del mercado inmobiliario a nivel de políticas, de inyección de capital y de estrategias de mercado en el desarrollo urbano de la ciudad de Managua, en lo que Brites (2017) ha denominado una producción neoliberal de la ciudad.

La inversión sobre los ejes viales que salen de forma radial desde el centro de la ciudad hacia los municipios aledaños se convierten en los espacio para estas inversiones selectivas, que de forma indirecta ha incidido en cambios en la relación funcional entre los centros urbanos, $\mathrm{y}$ específicamente entre estos y las áreas interurbanas de desarrollo casi exclusivamente habitacional.

Estos proyectos habitacionales como "innovaciones espaciales" que avanzan a través de la habilitación de tierra no urbanizada se encuentran al centro de este modelo de expansión, que avanza hacia zonas suburbanas hasta encontrarse con los bordes de las áreas urbanas más próximas. La problemática de este desarrollo es su indiferencia a la legislación urbana existente - que delimita los límites de expansión de la ciudad - y la tensión generada sobre los servicios e infraestructura urbana.

Este modelo de desarrollo resulta además en fenómenos de orden social como la tendencia al aislamiento y la formación de "islas" desasociadas del tejido social y urbano existente (Rodgers, 2006) mostrando un aumento de la segregación social y la fragmentación urbana a distintas escalas (Gutiérrez, 2020; Sánchez et al, 2017; López, 2016). Mientras que en el ámbito de lo económico la baja densidad y la expansión dispersa implican un aumento en los costos de gestión de la ciudad o al no existir la provisión de infraestructura y servicios, estos se convierten en costos directos para la población.

\section{Recomendaciones}

Las conclusiones expuestas dan apertura a una serie de interrogantes importantes, cuyo seguimiento permitiría dar continuidad a los hallazgos presentados. Antes de pensar en un proceso de institucionalización del área metropolitana, es indispensable cuestionar las cualidades del proceso de expansión en las líneas de:

- Evaluar el impacto social, económico y ambiental de la expansión urbana horizontal sobre zonas suburbanas y zonas de reserva natural. Adicionalmente, se pueden cuestionar estos procesos en relación con su indiferencia respecto a la normativa vigente y las implicaciones de esto.

- Analizar los modelos de gestión urbana de las ciudades que conformarían el área metropolitana, además de Managua, de forma que se pueda vislumbrar el modelo de formalización más óptimo llegado el momento.

- Desarrollar un análisis exhaustivo de las innovaciones espaciales que caracterizan la expansión urbana, más allá de los proyectos habitacionales, de forma que se pueda poner en relación las nuevas áreas residenciales con la aparición de áreas comerciales y ocio que comienzan a reconfigurar el patrón espacial urbano.

- Abrir nuevas líneas de investigación que indaguen sobre los procesos de mercado de suelo urbano y vivienda en la ciudad de Managua, que como se ha mostrado en este primer análisis, son el punto de partida de los procesos de reconfiguración y expansión urbana analizados. 
- Finalmente, ante la certeza de un proceso de expansión metropolitana disperso y desigual es necesario profundizar en la investigación sobre los patrones de crecimiento urbano de la ciudad de Managua, de forma que se pueda caracterizar a detalle la localización espacial de las innovaciones urbanas y sus cualidades (configuración espacial, socioeconómica, ambiental, etc.).

\section{Referencias}

Agencia de Cooperación Internacional del Japón JICA (2017). Plan Maestro para el Desarrollo Urbano del Municipio de Managua en la República de Nicaragua. Informe Final PARTE -I: Condiciones actuales. Disponible en https://openjicareport.jica.go.jp/pdf/12291662_01.pdf

Brites, W. (2017). La ciudad en la encrucijada neoliberal. Urbanismo mercado-céntrico y desigualdad socio-espacial en América Latina. Revista Braileira de Gestáo Urbana (Brazilian Journal of Urban Management, 9(3), 573-586. https://doi.org/10.1590/2175-3369.009.003.AO14

Ciccolella, P. (2012). Revisitando la metrópolis latinoamericana más allá de la globalización. En Revista Iberoamericana de Urbanismo, (8), 9-21. http://hdl.handle.net/2099/13012

CIDEU (s.f.). Aplicar el pensamiento estratégico concurrente a las conurbaciones.

Gutiérrez Lanza, D. (2020). El nuevo modelo de convivencia urbana: los enclaves habitacionales auto-segregados en Managua. Revista Arquitectura +, 5(10), 2-19. https://doi.org/10.5377/ arquitectura.v5i10.10558

Touraine, A. (sf.). La transformación de las metrópolis. Disponible en http://www.carlosmanzano. net/articulos/Touraine02.htm

López, N. (2016). Urbanización desigual en la ciudad de Managua, de 1995 a 2015 (documento de trabajo). http://biblioteca.clacso.edu.ar/clacso/becas/20160331030347/Informe.pdf

Rodgers, D. (2006). Desimbricando la ciudad: crimen, inseguridad y organización espacial en Managua, Nicaragua. Encuentro. (73), 8-24.

Rodríguez, A., \& Abramo, P. (2005). Grandes proyectos urbanos y su impacto en el mercado del suelo urbano.

Rojas, E. (2005). Las regiones metropolitanas en América Latina. Problemas de gobierno y desarrollo. En Rojas, E., Cuadrado-Roura, J. \& Fernández, J. (2005) (Eds.). Gobernar las metrópolis.

Tella, G. \& Robledo, L. (2011), “Áreas Metropolitanas. La necesidad de coordinar políticas. Fortalezas y debilidades de diferentes políticas". En: Revista Mercado y Empresas para Servicios Públicos Nro. 63 (agosto), Ed. IC Argentina, pp. 34-45. https://www.guillermotella.com/articulos/la-gestion-metropolitana-diez-estrategias-de-actuacion/

Tomás, M. (2016). Tendencias metropolitanas en el mundo. https://www.metropolis.org/sites/default/files/metropolis-observatory_issue-paper-1_es

World Bank, Augustin, M., Acero, J.L., Aguilera, A. \& García Lozano, M. (eds.) (2017). Central America Urbanization Review: Making Cities Work for Central America. Directions in Development. Washington, DC: World Bank. https://doi.org/10.1596/978-1-4648-0985-9 